\title{
ON HAHN-BANACH TYPE THEOREMS FOR HILBERT C*-MODULES
}

\author{
MICHAEL FRANK
}

\begin{abstract}
We show three Hahn-Banach type extension criteria for (sets of) bounded $\mathrm{C}^{*}$-linear maps of Hilbert $\mathrm{C}^{*}$-modules to the underlying $\mathrm{C}^{*}$-algebras of coefficients. One criterion establishes an alternative description of the property of $\mathrm{C}^{*}$-algebras to be monotone complete or additively complete.
\end{abstract}

\section{INTRODUCTION}

The Hahn-Banach theorem belongs to the central results of Banach space theory, together with the open mapping theorem, the closed graph theorem, and others. When investigating the generalized Hahn-Banach extension problem for Banach $\mathrm{C}^{*}$-modules, their $\mathrm{C}^{*}$-submodules and bounded $\mathrm{C}^{*}$-linear maps thereof to the $\mathrm{C}^{*}$ algebra of coefficients, we must resort to case studies since a general answer is out of reach at present. The goal of the present paper is to find criteria for the particular class of Hilbert $\mathrm{C}^{*}$-modules, i.e. of Banach modules over $\mathrm{C}^{*}$-algebras $A$ that admit an $A$-valued inner product, [22, 18]. We aim to preserve the $\mathrm{C}^{*}$-algebra of coefficients as the codomain of the extended maps.

In comparison to the particular case of Hilbert spaces the problem is non-trivial since Hilbert $\mathrm{C}^{*}$-submodules of Hilbert $\mathrm{C}^{*}$-modules are in general not direct summands, and bounded module maps of $\mathrm{C}^{*}$-submodules to the $\mathrm{C}^{*}$-algebra of coefficients may be non-extendable to bounded module maps on the entire Hilbert $\mathrm{C}^{*}$-module. To give an example, let $A=\mathrm{C}([0,1])$ denote the $\mathrm{C}^{*}$-algebra of all continuous functions on the unit interval $[0,1]$ and $I=\mathrm{C}_{0}((0,1])$ the subset of all continuous functions vanishing at zero. The corresponding $A$-valued inner product is defined by $\langle f, g\rangle_{A}=f g^{*}$ for $f, g \in A$. The map $f \in I \longrightarrow g(t)=\sin (1 / t) \cdot f(t) \in I$, $t \in[0,1]$, is a bounded module map on $I$ which cannot be continued to a bounded $A$-linear map on $A$ preserving the codomain $\mathrm{C}^{*}$-algebra $A$. Moreover, the property of a Hilbert $\mathrm{C}^{*}$-submodule $\mathcal{N}$ of a Hilbert $\mathrm{C}^{*}$-module $\mathcal{M}$ of being an orthogonal direct summand thereof sometimes depends on the choice of the $\mathrm{C}^{*}$-valued inner product on $\mathcal{M}$, even if the derived norms are equivalent, [9]. In other words, we are faced by a difference between the properties of $\mathrm{C}^{*}$-submodules of being an orthogonal or merely a topological direct summand, cf. Example 3.4 .

Hahn-Banach extension problems for bounded module maps have several origins and applications. For Hilbert $\mathrm{C}^{*}$-modules the problem to extend bounded module maps was first touched in W. L. Paschke's papers [22, 23]. Knowing that the analogue of Riesz representation theorem for bounded module functionals may fail, in general, W. L. Paschke analyzed the conditions under which the $A$-valued inner

1991 Mathematics Subject Classification. Primary 46L08; Secondary 46L35, 46L07, 47L25.

Key words and phrases. Hilbert $\mathrm{C}^{*}$-module, bounded module map, Hahn-Banach theorem, completely bounded module map, operator spaces, operator modules. 
product on a Hilbert $A$-module $\{\mathcal{M},\langle.,\rangle$.$\} over a fixed \mathrm{C}^{*}$-algebra $A$ can be continued to the Banach $A$-module $\mathcal{M}^{\prime}$ of all bounded $A$-linear maps $r: \mathcal{M} \rightarrow A$ turning it into a Hilbert $A$-module, among other similar extension problems. A particular reinterpretation of this problem is to ask for the existence of isometric extension of bounded module maps $r$ on $\mathcal{M}$ to those on $\mathcal{M}^{\prime}$ preserving the values of $r$ if it is restricted to the canonically isometrically embedded copy of $\mathcal{M}$ in $\mathcal{M}^{\prime}$. Later on H. Lin [20], M. Hamana [13] and the author [5, 6] continued the study of extension problems of bounded module maps and $\mathrm{C}^{*}$-valued inner products on Hilbert $\mathrm{C}^{*}$-modules and realized the key role played by the order structure of the positive cone of the underlying $\mathrm{C}^{*}$-algebra of coefficients. In particular, these results mark the departure from a topological point of view on Hahn-Banach extension problems for bounded module maps on pairs of Hilbert $\mathrm{C}^{*}$-modules since there exists a commutative $\mathrm{AW}^{*}$-algebra (15]) described by E. E. Floyd [3] for which there does not exist any Frechét topology with respect to which both the partial order structure of its positive cone (which is monotone complete) and its linear structure are continuous at once. The reader is encouraged to consult the publications 13 , 5 , 6 and, especially, to study H. Lin's work [20] for more detailed information on the achieved module map extension results and their proofs. We refer to them at the appropriate places of the present paper.

A second root and motivation have been results by G. Vincent-Smith [31, G. Wittstock [33, 34, Ching-Yun Suen [30], P. S. Muhly and Qiyuan Na [21], A. M. Sinclair and R. R. Smith [29] on Hahn-Banach type theorems for completely bounded module maps on matricial normed Banach $\mathrm{C}^{*}$-(bi-)modules (i.e. operator (bi-)modules over $\mathrm{C}^{*}$-algebras) if the range of the extensions is allowed to grow inside a (larger) injective $\mathrm{C}^{*}$-algebra, for example the injective envelope of the $\mathrm{C}^{*}$ algebra of coefficients $(\sqrt{12})$ ). Hilbert $\mathrm{C}^{*}$-modules have the advantage that they can always be represented as operator modules on Hilbert spaces, and that bounded $\mathrm{C}^{*}$ linear maps between them are automatically completely bounded, 34, Prop. 2.7, 2.8]. We give more details on this point of view in the last section.

To formulate the Hahn-Banach type criteria we obtained the reader should be aware that a Hilbert $A$-module $\{\mathcal{M},\langle.,\rangle$.$\} , its A$-dual Banach $A$-module $\mathcal{M}^{\prime}$ and its $A$-bidual Banach $A$-module $\mathcal{M}^{\prime \prime}$ are connected by two canonical isometric embeddings $\mathcal{M} \hookrightarrow \mathcal{M}^{\prime \prime} \hookrightarrow \mathcal{M}^{\prime}$, where surjectivity may fail at any position and any possible interrelation may be realized by examples, cf. [22, 23]. Moreover, the process taking $A$-duals stabilizes since the $A$-dual of $\mathcal{M}^{\prime \prime}$ equals the $A$-dual of $\mathcal{M}$. Also, the $A$-valued inner product on $\mathcal{M}$ can be always continued to an $A$-valued inner product on $\mathcal{M}^{\prime \prime}$, cf. [23, Th. 2.4]. To define some properties of $\mathrm{C}^{*}$-algebras that come into the play we say that a $\mathrm{C}^{*}$-algebra $A$ is monotone complete if every bounded increasingly directed net $\left\{a_{\alpha}: \alpha \in I\right\}$ of self-adjoint elements of $A$ has a least upper bound $a=\sup \left\{a_{\alpha}: \alpha \in I\right\}$ inside $A$. It is said to be additively complete if every norm-bounded infinite sum of positive elements possesses a least upper bound inside $A$. For more details we refer to the next section were the preliminaries will be explained.

Theorem 1.1. For $C^{*}$-algebras $A$ the following conditions are equivalent:

(i) for any pair $\{\{\mathcal{M},\langle.,\rangle\},. \mathcal{N} \subseteq \mathcal{M}\}$ of a Hilbert $A$-module and a Hilbert $A$ submodule there exists an A-linear isometric embedding $\phi$ of the Banach A-module $\mathcal{N}^{\prime}$ into the Banach $A$-module $\mathcal{M}^{\prime}$ extending the canonical embedding of $\mathcal{N}$ into $\mathcal{M}^{\prime}$ induced by the A-valued inner product. 
(ii) for any pair $\{\{\mathcal{M},\langle.,\rangle\},. \mathcal{N} \subseteq \mathcal{M}\}$ of a Hilbert $A$-module and a Hilbert $A$-submodule every bounded A-linear mapping $r: \mathcal{N} \rightarrow A$ can be continued to a bounded A-linear mapping $r^{\prime}: \mathcal{M} \rightarrow A$ so that (a) $\|r\|=\left\|r^{\prime}\right\|$, (b) $r^{\prime}$ restricted to $\mathcal{N}$ equals $r$ and $(c) r^{\prime}$ restricted to $\mathcal{N}^{\perp} \subseteq \mathcal{M}$ equals zero.

(iii) the multiplier $C^{*}$-algebra $M(A)$ of $A$ is monotone complete.

(iv) the multiplier $C^{*}$-algebra $M(A)$ of $A$ is additively complete.

In this case the $C^{*}$-algebra $A$ has the property that the biorthogonal complements $\mathcal{N}^{\perp \perp}$ of Hilbert $A$-submodules $\mathcal{N}$ of an arbitrary Hilbert $A$-module $\mathcal{M}$ estimated with respect to the $A$-bidual Hilbert $A$-module $\mathcal{M}^{\prime \prime}$ of $\mathcal{M}$ are orthogonal direct summands of $\mathcal{M}^{\prime \prime}$ automatically.

The implications (iii) $\rightarrow$ (ii) and (iii) $\rightarrow$ (i) have been established by Huaxin Lin 20, Th. 3.8]. The equivalence (iii) $\leftrightarrow$ (iv) has been discovered by K. Saitô and J. D. M. Wright [28, §3]. The goal of the present paper is the equivalence (i) $\leftrightarrow$ (ii) and the implication (i)/(ii) $\rightarrow$ (iii)/(iv). During the course of proof, we obtain a result valid for particular pairs of some Hilbert $\mathrm{C}^{*}$-module and one of its Hilbert $\mathrm{C}^{*}$-submodules without any restrictions to the properties of the $\mathrm{C}^{*}$-algebra of coefficients:

Theorem 1.2. Let $A$ be a $C^{*}$-algebra, $\{\mathcal{M},\langle.,\rangle$.$\} be a Hilbert A$-module and $\mathcal{N} \subseteq \mathcal{M}$ one of its Hilbert $A$-submodules.

Then every bounded A-linear mapping $r: \mathcal{N} \rightarrow A$ can be continued to a bounded A-linear mapping $r^{\prime}: \mathcal{M} \rightarrow A$ so that (a) $\|r\|=\left\|r^{\prime}\right\|$, (b) $r^{\prime}$ restricted to $\mathcal{N}$ equals $r$ and (c) the extended mappings of $\mathcal{N}^{\prime}$ form a Banach A-submodule of $\mathcal{M}^{\prime}$, and (d) the extensions $\left\{r_{n}^{\prime}: n \in \mathcal{N}\right\}$ of the standardly embedded mappings $\left\{r_{n}=\langle., n\rangle: n \in \mathcal{N}\right\} \subseteq \mathcal{M}^{\prime}$ coincide with the latter, if and only if every bounded A-linear mapping $r: \mathcal{N} \rightarrow A$ can be continued to a bounded A-linear mapping $r^{\prime}: \mathcal{M} \rightarrow A$ so that (a) $\|r\|=\left\|r^{\prime}\right\|$, (b) $r^{\prime}$ restricted to $\mathcal{N}$ equals $r$ and (c) $r^{\prime}$ restricted to $\mathcal{N}^{\perp} \subseteq \mathcal{M}$ equals zero.

This happens if and only if the A-bidual Hilbert A-module $\mathcal{M}^{\prime \prime}$ of $\mathcal{M}$ is the orthogonal direct sum of the orthogonal and the biorthogonal complement of $\mathcal{N}$ inside $\mathcal{M}^{\prime \prime}$ with respect to the continued A-valued inner product $\langle.,$.$\rangle , i.e.$ $\mathcal{M}^{\prime \prime}=\mathcal{N}^{\perp \perp} \oplus \mathcal{N}^{\perp}$. Moreover, the A-bidual Banach A-modules $\mathcal{N}^{\prime \prime}$ and $\left(\mathcal{N}^{\perp \perp}\right)^{\prime \prime}$ coincide isometrically.

To weaken the additional assumption on the embedding $\phi: \mathcal{N}^{\prime} \rightarrow \mathcal{M}^{\prime}$ to be isometric we can suppose that it has to be merely bounded and $A$-linear with closed range, cf. Example 3.4. Going with these assumptions we can formulate the following result:

Theorem 1.3. Let $A$ be a $C^{*}$-algebra, $\{\mathcal{M},\langle.,\rangle$.$\} be an A-reflexive Hilbert A$ module, i.e. $\mathcal{M}=\mathcal{M}^{\prime \prime}$. Then for Hilbert A-submodules $\mathcal{N} \subseteq \mathcal{M}$ the following two conditions are equivalent:

(i) $\mathcal{N}$ is a topological direct summand of $\mathcal{M}$ (i.e. not necessarily an orthogonal direct summand).

(ii) $\mathcal{N} \equiv \mathcal{N}^{\perp \perp} \subseteq \mathcal{M}$ and there exists a bounded A-linear injective mapping $\phi: \mathcal{N}^{\prime} \rightarrow \mathcal{M}^{\prime}$ such that $\phi(r)[n]=r(n)$ for every $r \in \mathcal{N}^{\prime}$, every $n \in \mathcal{N}$.

Without the assumption of $\mathcal{M}$ being A-reflexive (ii) does not imply (i), in general.

The next section contains some definitions and facts from the literature that have to be stated for proving. The third section is concerned with metric aspects of 
the proof, whereas the forth section deals with its inner product aspects. The proof of the theorems is divided into a number of propositions which are of independent interest. We close the considerations with a remark on the structure of particular extensions of $\mathrm{C}^{*}$-valued functionals on Hilbert $\mathrm{C}^{*}$-modules and on the relation of our results to Hahn-Banach type problems for operator modules.

\section{Preliminaries}

In this section we give definitions and basic facts about Hilbert $\mathrm{C}^{*}$-modules and $\mathrm{C}^{*}$-algebras necessary for the proofs of the theorems. The papers $22,16,0,19,20$, 9], some chapters in [14, 32], and the books by E. C. Lance [18 and by I. Raeburn and D. P. Williams [27] are used as standard sources of reference on Hilbert $\mathrm{C}^{*}$ module theory. We make the convention that all $\mathrm{C}^{*}$-modules of the present paper are left modules by definition. A pre-Hilbert $A$-module over a $C^{*}$-algebra $A$ is an $A$-module $\mathcal{M}$ equipped with an $A$-valued mapping $\langle.,\rangle:. \mathcal{M} \times \mathcal{M} \rightarrow A$ which is $A$-linear in the first argument and has the properties:

$$
\langle x, y\rangle=\langle y, x\rangle^{*},\langle x, x\rangle \geq 0 \quad \text { with equality iff } \quad x=0 .
$$

The mapping $\langle.,$.$\rangle is said to be the A-valued inner product on \mathcal{M}$. A pre-Hilbert $A$-module $\{\mathcal{M},\langle.,\rangle$.$\} is Hilbert if and only if it is complete with respect to the$ norm $\|\|=.\|\langle., .\rangle\|_{A}^{1 / 2}$. We always assume that the linear structures of $A$ and $\mathcal{M}$ are compatible. Two Hilbert $A$-modules are isomorphic if they are isometrically isomorphic as Banach $A$-modules, if and only if they are unitarily isomorphic, [18]. Moreover, Banach $A$-modules can carry unitarily non-isomorphic $A$-valued inner products that induce equivalent norms to the given one, nevertheless, [9].

A Hilbert $A$-module $\mathcal{M}$ is full if the norm-closed linear span $\langle\mathcal{M}, \mathcal{M}\rangle$ of the values of the $A$-valued inner product inside $A$ coincides with $A$. A Hilbert $A$ module $\{\mathcal{M},\langle.,\rangle$.$\} over a \mathrm{C}^{*}$-algebra $A$ is said to be self-dual if and only if every bounded module map $r: \mathcal{M} \rightarrow A$ is of the form $\left\langle., x_{r}\right\rangle$ for some element $x_{r} \in \mathcal{M}$. The set of all bounded module maps $r: \mathcal{M} \longrightarrow A$ forms a Banach $A$-module $\mathcal{M}^{\prime}$. A Hilbert $A$-module is called $C^{*}$-reflexive (or more precisely, $A$-reflexive) if and only if the map $\Omega$ defined by the formula $\Omega(x)[r]=r(x)$ for each $x \in \mathcal{M}$, every $r \in \mathcal{M}^{\prime}$, is a surjective module mapping of $\mathcal{M}$ onto the Banach $A$-module $\mathcal{M}^{\prime \prime}$, where $\mathcal{M}^{\prime \prime}$ consists of all bounded module maps from $\mathcal{M}^{\prime}$ to $A$. Note that the property of being self-dual does not depend on the choice of the $\mathrm{C}^{*}$-algebra of coefficients $A$ within $\langle\mathcal{M}, \mathcal{M}\rangle \subseteq A \subseteq M(\langle\mathcal{M}, \mathcal{M}\rangle)$, whereas the property of being $A$-reflexive sometimes does. As an example consider the $\mathrm{C}^{*}$-algebra $A=c_{0}$ of all sequences converging to zero and set $\mathcal{M}=c_{0}$ with the standard $A$-valued inner product. The multiplier $\mathrm{C}^{*}$-algebra of $A=c_{0}$ is $M(A)=l_{\infty}$, the set of all bounded sequences. Then $\mathcal{M}^{\prime}$ equals $l_{\infty}$ as a one-sided $A$-module, whereas $\mathcal{M}^{\prime \prime}=c_{0}$ again. In contrast, the set of all bounded $M(A)$-linear maps of $\mathcal{M}^{\prime}$ to $M(A)$ can be identified with $l_{\infty}$. Generally speaking, the $A$-dual Banach $A$-module $\mathcal{M}^{\prime}$ of a Hilbert $A$-module $\mathcal{M}$ can be described as the linear hull of the completed with respect to the topology $\left\{\|\langle x, .\rangle\|_{A}: x \in \mathcal{M},\|x\| \leq 1\right\}$ unit ball of $\mathcal{M}$, [9, Th. 6.4].

During our considerations we need a decomposition property of elements of Hilbert $A$-modules $\mathcal{M}$ over $\mathrm{C}^{*}$-algebras $A$ that is sharper than any density argument of $A \circ \mathcal{M}$ in $\mathcal{M}$ usually given, cf. [18, p. 5]. It could be attributed to a number of mathematicians like E. Hewitt, B. E. Johnson, A. M. Sinclair, G. Skandalis, and others. We cite a version which may be found in [26, Th. 4.1] for example. 
Lemma 2.1. Let $A$ be a $C^{*}$-algebra acting on a Banach $A$-module $\mathcal{M}$ in such a way that $A \circ \mathcal{M}$ is norm-dense in $\mathcal{M}$. Then every element $x \in \mathcal{M}$ can be decomposed as $x=$ ay for some $y \in \mathcal{M}$ and some positive $a \in A$ with $\|a\| \leq 1$. Moreover, for any given $\varepsilon>0$ an estimate $\|x-y\|<\varepsilon$ can be observed.

If $\mathcal{M}$ is a Hilbert $A$-module then by construction the element a can be selected from the smallest norm-closed two-sided ideal of $A$ containing the value $\langle x, x\rangle$.

As an immediate consequence we obtain that any bounded $A$-linear or $M(A)$ linear map from $\mathcal{M}$ into $M(A)$ has a range inside $A \subseteq M(A)$. At the same time every $A$-linear map is automatically $M(A)$-linear since $M(A)$ is the strict completion of $A$ and the considered maps are continuous. The $A$-valued inner product on a Hilbert $A$-module $\mathcal{M}$ can always be lifted to an $A$-valued inner product on its $A$-bidual Banach $A$-module $\mathcal{M}^{\prime \prime}$, 23, Th. 2.4]. We already mentioned the canonical isometric embeddings $\mathcal{M} \hookrightarrow \mathcal{M}^{\prime \prime} \hookrightarrow \mathcal{M}^{\prime}$ arising from the $\mathrm{C}^{*}$-valued inner product, and the coincidence $(\mathcal{M})^{\prime} \equiv\left(\mathcal{M}^{\prime \prime}\right)^{\prime}$, [23]. A Hilbert $A$-module $\mathcal{M}$ is said to be orthogonally complementary as a Hilbert $A$-module if each Hilbert $A$-module $\mathcal{L}$ admitting a bicontinuous embedding of $\mathcal{M}$ as a Banach $A$-submodule is orthogonally decomposable as $\mathcal{L}=\mathcal{M} \oplus \mathcal{M}^{\perp}$.

Proposition 2.2. (cf. [20, Th. 2.9], [9, Th. 5.6, 6.5]) Let $A$ be a $C^{*}$-algebra and $\{\mathcal{M},\langle.,\rangle$.$\} be a full Hilbert A-module. Then every$ bounded module operator on $\mathcal{M}$ possesses an adjoint bounded module operator if and only if $\mathcal{M}$ is orthogonally complementary. The restriction on $\mathcal{M}$ of being full cannot be avoided. In this situation either $\mathcal{M}$ is self-dual or, firstly, the Avalued inner product $\langle.,$.$\rangle can be extended to an M(A)$-valued inner product on $\mathcal{M}^{\prime}$ and, secondly, every bounded A-linear operator on $\mathcal{M}^{\prime}$ preserves the Banach A-submodule $\mathcal{M} \hookrightarrow\{\langle., x\rangle: x \in \mathcal{M}\} \subseteq \mathcal{M}^{\prime}$ invariant.

The next statement characterizes monotone complete and additively complete $\mathrm{C}^{*}$-algebras by alternative conditions. For example, $\mathrm{W}^{*}$-algebras and commutative $\mathrm{C}^{*}$-algebras $A=\mathrm{C}(X)$ with stonean compact spaces $X$ are monotone complete, cf. 115, 20, 13, 5 .

Proposition 2.3. (汭, Prop. 4.7], [13, Th. 2.2], [20, Lemma 3.7], [28, §3]) $A C^{*}$-algebra $A$ is monotone complete if and only if $A$ is additively complete, if and only if for every Hilbert $A$-module $\{\mathcal{M},\langle.,\rangle$.$\} the A-valued inner product \langle.,$. on $\mathcal{M}$ can be continued to an A-valued inner product $\langle., .\rangle_{D}$ on the A-dual Banach $A$-module $\mathcal{M}^{\prime}$ turning $\left\{\mathcal{M}^{\prime},\langle., .\rangle_{D}\right\}$ into a self-dual Hilbert $A$-module.

Moreover, in this situation the equalities $\langle x, y\rangle_{D}=\langle x, y\rangle,\langle x, r\rangle_{D}=r(x)$ are satisfied for every $x, y \in \mathcal{M} \hookrightarrow \mathcal{M}^{\prime}$, every $r \in \mathcal{M}^{\prime}$.

As a proving technique we need a basic construction to switch from a given Hilbert $A$-module $\mathcal{M}$ to a bigger Hilbert $A^{* *}$-module $\mathcal{M}^{\#}$ while preserving many useful properties and guaranteeing the existence and uniqueness of extended operators and $A-\left(A^{* *}-\right)$ valued inner products, (cf. H. Lin [19, Def. 1.3], [22, §4]). For example, the $\mathrm{C}^{*}$-valued inner product on a Hilbert $\mathrm{W}^{*}$-module $\mathcal{M}$ over a $\mathrm{W}^{*}$ algebra $B$ can always be continued to an $\mathrm{C}^{*}$-valued inner product on its $\mathrm{C}^{*}$-dual Banach $W^{*}$-module $\mathcal{M}^{\prime}$ turning it into a self-dual Hilbert $\mathrm{W}^{*}$-module, [22, Th. 3.2]. Therefore, for Hilbert $W^{*}$-modules we have $\mathcal{M}^{\prime \prime} \equiv \mathcal{M}^{\prime}$. Moreover, taking the biorthogonal complement $\mathcal{N}^{\perp \perp}$ of a $\mathrm{C}^{*}$-submodule $\mathcal{N}$ of a self-dual Hilbert $\mathrm{W}^{*}$ module $\mathcal{M}$ and forming its $\mathrm{C}^{*}$-dual Banach $\mathrm{W}^{*}$-module $\mathcal{N}^{\prime}$ gives the same subset of $\mathcal{M}$ under the canonical indentifications usually made. Also, the $\mathrm{C}^{*}$-linear hull 
of the completed with respect to the topology $\left\{|f(\langle., x\rangle)|: x \in \mathcal{N}, f \in B_{*}\right\}$ unit ball of $\mathcal{N}$ may be identified with both $\mathcal{N}^{\perp \perp}$ and $\mathcal{N}^{\prime}$ inside $\mathcal{M}$ in this situation, [4, Th. 3.2]. We refer to [22, 19, 1, 4 for more details.

Let $\{\mathcal{M},\langle.,\rangle$.$\} be a left pre-Hilbert A$-module over a fixed $\mathrm{C}^{*}$-algebra $A$. The algebraic tensor product $A^{* *} \otimes \mathcal{M}$ becomes a left $A^{* *}$-module defining the action of $A^{* *}$ on its elementary tensors by the formula $a b \otimes h=a(b \otimes h)$ for $a, b \in A^{* *}$, $h \in \mathcal{M}$. Now, setting

$$
\left[\sum_{i} a_{i} \otimes h_{i}, \sum_{j} b_{j} \otimes g_{j}\right]=\sum_{i, j} a_{i}\left\langle h_{i}, g_{j}\right\rangle b_{j}^{*}
$$

on finite sums of elementary tensors, a degenerate $A^{* *}$-valued inner pre-product is defined. Factorizing $A^{* *} \otimes \mathcal{M}$ by $N=\left\{z \in A^{* *} \otimes \mathcal{M}:[z, z]=0\right\}$ we obtain a pre-Hilbert $A^{* *}$-module denoted by $\mathcal{M}^{\#}$ in the sequel. It contains $\mathcal{M}$ as an $A$ submodule. If $\mathcal{M}$ is Hilbert, then $\mathcal{M}^{\#}$ is Hilbert, and vice versa. More difficult is the transfer of self-duality. If $\mathcal{M}$ is self-dual, then $\mathcal{M}^{\#}$ is self-dual, too, but to demonstrate the converse is an open problem. Other standard properties like e.g. $C^{*}$-reflexivity cannot be transferred. Every bounded $A$-linear operator $T$ on $\mathcal{M}$ possesses a unique extension to a bounded $A^{* *}$-linear operator on $\mathcal{M}^{\#}$ preserving the operator norm. Note that the relation $S_{1} \perp S_{2}$ for subsets $S_{1}, S_{2} \subseteq \mathcal{M}$ gives rise to the relation $S_{1}^{\#} \perp S_{2}^{\#}$ for the corresponding subsets of $\mathcal{M}^{\#}$.

Lemma 2.4. Let $A$ be a $C^{*}$-algebra and $\mathcal{M}, \mathcal{N}$ be two Hilbert A-modules. The kernel $\operatorname{ker}(T)$ of every bounded A-linear operator $T: \mathcal{M} \rightarrow \mathcal{N}$ coincides with its biorthogonal complement with respect to $\mathcal{M}$. In particular, the kernel of every bounded A-linear map $r: \mathcal{M} \rightarrow A$ has this property. If for some element $r \in \mathcal{M}^{\prime}$ its kernel possesses a trivial orthogonal complement in $\mathcal{M}$, then $r$ is the zero mapping.

Proof. Assume $\operatorname{ker}(T) \neq \operatorname{ker}^{\perp \perp}(T)$ inside of $\mathcal{M}$. Form the Hilbert $A$-module $\mathcal{L}=$ $\mathcal{M} \oplus \mathcal{N}$ and consider $T$ as an operator on $\mathcal{L}$ with $\operatorname{kernel} \operatorname{ker}(T) \oplus \mathcal{N}$. Consider the unique extension of $T$ to a bounded $A^{* *}$-linear operator on the self-dual Hilbert $A^{* *}$-module $\left(\mathcal{L}^{\#}\right)^{\prime}$, also denoted by $T$. Taking advantage of the special properties of Hilbert $\mathrm{W}^{*}$-modules and of the extension operation \# as described above we obtain that both $\operatorname{ker}(T)^{\#}$ and $\left(\operatorname{ker}(T)^{\perp \perp}\right)^{\#}$ are contained in the biorthogonal complement of $\operatorname{ker}(T) \subseteq \mathcal{M} \hookrightarrow\left(\mathcal{L}^{\#}\right)^{\prime}$ estimated with respect to $\left(\mathcal{L}^{\#}\right)^{\prime}$. What is more, the latter is part of the kernel of the extended to $\left(\mathcal{L}^{\#}\right)^{\prime}$ operator $T$ since $T$ becomes adjointable there and admits a polar decomposition, [32, Prop. 15.3.7] and [22]. We arrive at a contradiction.

Since Banach $\mathrm{C}^{*}$-modules may admit different $\mathrm{C}^{*}$-valued inner products that induce equivalent norms to the given one and, nevertheless, are not unitarily equivalent (cf. [9]) one further comment is necessary: the arguments do not depend on the choice of the $A$-valued inner products on $\mathcal{M}$ and $\mathcal{N}$ within their respective unitary equivalence classes since on $\left(\mathcal{L}^{\#}\right)^{\prime}$ any two $A^{* *}$-valued inner products are unitarily equivalent by self-duality and the kernel of $T$ is always an orthogonal direct summand there, cf. [22].

\section{The Metric AspeCts of the PROOF}

This section is devoted to the various Banach $\mathrm{C}^{*}$-modules that are related to a pair $\{\mathcal{M}, \mathcal{N} \subseteq \mathcal{M}\}$ consisting of a Hilbert $\mathrm{C}^{*}$-module $\{\mathcal{M},\langle.,\rangle$.$\} and one of its$ Hilbert $\mathrm{C}^{*}$-submodules $\mathcal{N}$, and to their interrelation. The main emphasis is put on 
pairwise containment relations, isomorphisms and direct sum decompositions. The orthogonality of $\mathrm{C}^{*}$-submodules is merely used for their definition and for their identification with other $\mathrm{C}^{*}$-submodules. We start with two simple observations concerning $\mathrm{C}^{*}$-reflexive Hilbert $\mathrm{C}^{*}$-modules and their properties.

Lemma 3.1. Let $A$ be a $C^{*}$-algebra and $\{\mathcal{M}\langle.,\rangle$.$\} be an A$-reflexive, non-self-dual Hilbert $A$-module. Then $\mathcal{M}$ is the largest Banach $A$-submodule $\mathcal{L} \subseteq \mathcal{M}^{\prime}$ which is a Hilbert A-module itself, contains the canonical copy of $\mathcal{M}$ in $\mathcal{M}^{\prime}$ as a Hilbert A-submodule and possesses the same A-dual Banach A-module $\mathcal{M}^{\prime}$.

That is, if there exists a Banach A-submodule $\mathcal{L} \subseteq \mathcal{M}^{\prime}$ which is a Hilbert $A$ module itself, contains the canonical copy of $\mathcal{M}$ in $\overline{\mathcal{M}}^{\prime}$ as a Hilbert $A$-submodule and is larger than $\mathcal{M}$, then there exists a bounded A-linear mapping $r: \mathcal{M} \rightarrow A$ that cannot be continued to a bounded A-linear mapping $r^{\prime}: \mathcal{L} \rightarrow A$.

Proof. Let $\mathcal{L}$ be a Banach $A$-submodule of $\mathcal{M}^{\prime}$ with the properties requested. Consider the map from $\mathcal{L}^{\prime}$ into $\mathcal{M}^{\prime}$ defined by the restriction of every element of $\mathcal{L}^{\prime}$ to the canonical copy of $\mathcal{M}$ in $\mathcal{M}^{\prime}$ which has been supposed to be also contained in $\mathcal{L}$. This map defines an isometric embedding of $\mathcal{L}^{\prime}$ into $\mathcal{M}^{\prime}$, [23, Prop. 2.1]. If this map is onto then $\mathcal{L}$ has to coincide with the canonically embedded copy of $\mathcal{M}$ in $\mathcal{M}^{\prime}$ since $\mathcal{M}$ was supposed to be $\mathrm{C}^{*}$-reflexive.

Lemma 3.2. Let $A$ be a $C^{*}$-algebra, $\{\mathcal{M},<., .>\}$ be an A-reflexive Hilbert $A$ module and $\mathcal{N} \subseteq \mathcal{M}$ be one of its Hilbert $A$-submodules. Then the biorthogonal complement $\mathcal{N}^{\perp \perp}$ of $\mathcal{N}$ with respect to $\mathcal{M}$ is A-reflexive, too, and $\mathcal{N}^{\perp \perp} \cong\left(\mathcal{N}^{\perp \perp}\right)^{\prime \prime}$. In general, $\mathcal{N}^{\prime \prime}$ may be smaller than $\left(\mathcal{N}^{\perp \perp}\right)^{\prime \prime}$.

Proof. For every element $p \in \mathcal{M}^{\prime}$ we obtain a unique element $r_{p} \in\left(\mathcal{N}^{\perp \perp}\right)^{\prime}$ by restricting $p$ to $\mathcal{N}^{\perp \perp} \subseteq \mathcal{M}$. The resulting map from $\mathcal{M}^{\prime}$ into $\left(\mathcal{N}^{\perp \perp}\right)^{\prime}$ is a contractive $A$-linear mapping of Banach $A$-modules. By Lemma 2.4 the kernel of it consists of all elements of $\mathcal{M}^{\prime}$ vanishing on $\mathcal{N}$. Now, define a map $\pi:\left(\mathcal{N}^{\perp \perp}\right)^{\prime \prime} \rightarrow \mathcal{M}^{\prime \prime} \equiv \mathcal{M}$ by the rule $\pi(r)(p):=r\left(r_{p}\right)$ for $r \in\left(\mathcal{N}^{\perp \perp}\right)^{\prime \prime}, p \in \mathcal{M}^{\prime}$. It is obviously a module map, and $\pi\left(\left(\mathcal{N}^{\perp \perp}\right)^{\prime \prime}\right)$ is contained in the biorthogonal complement of $\mathcal{N}$ with respect to $\mathcal{M}$ by construction. Since $\mathcal{M}$ is $\mathrm{C}^{*}$-reflexive and $\mathcal{N}^{\perp \perp}$ is a Hilbert $\mathrm{C}^{*}$-submodule of $\mathcal{M}$ we have the equality $\|\pi(r)\|_{\mathcal{N}^{\perp \perp}}=\|\pi(r)\|_{\mathcal{M}^{\prime \prime}}$. However, $\pi(r)$ acts on $\mathcal{N}^{\perp \perp} \hookrightarrow$ $\mathcal{M}^{\prime}$ as $r$ acts on $\mathcal{N}^{\perp \perp} \hookrightarrow\left(\mathcal{N}^{\perp \perp}\right)^{\prime}$, and therefore $\|\pi(r)\|_{\mathcal{N} \perp \perp}=\|r\|_{(\mathcal{N} \perp \perp)^{\prime \prime}}$ for any $r \in\left(\mathcal{N}^{\perp \perp}\right)^{\prime \prime}$. So the map $\pi$ is isometric. Moreover, the map $\pi$ is onto since $\mathcal{N}^{\perp \perp} \subseteq\left(\mathcal{N}^{\perp \perp}\right)^{\prime \prime}$ is fixed under $\pi$.

The isometric inclusion $\mathcal{N}^{\prime \prime} \hookrightarrow\left(\mathcal{N}^{\perp \perp}\right)^{\prime \prime} \equiv \mathcal{N}^{\perp \perp}$ follows from Lemma 3.1 since $\mathcal{N}^{\prime} \supseteq\left(\mathcal{N}^{\perp \perp}\right)^{\prime}$ by Lemma 2.1, $\mathcal{N} \subseteq \mathcal{N}^{\perp \perp} \hookrightarrow \mathcal{N}^{\prime}$ isometrically and Lemma 3.1 is valid. However, $\mathcal{N}$ need not to be equal to $\mathcal{N}^{\perp \perp}$ in $\mathcal{M}$. To give an example set $A=\mathcal{M}=\mathrm{C}([0,1])$ and $\mathcal{N}=\mathrm{C}_{0}((0,1])$ to obtain $\mathcal{N}^{\prime \prime} \equiv \mathcal{N} \subsetneq \mathcal{N}^{\perp \perp} \equiv\left(\mathcal{N}^{\perp \perp}\right)^{\prime \prime} \equiv$ $\mathcal{M} \equiv \mathcal{M}^{\prime \prime}$.

The next step is to prepare the proofs of the first part of Theorem 1.2 and to demonstrate Theorem 1.3. We will do this by characterizing some special geometric properties of isometric $A$-linear embeddings $\phi: \mathcal{N}^{\prime} \rightarrow \mathcal{M}^{\prime}$ for pairs consisting of a Hilbert $A$-module $\{\mathcal{M},\langle.,\rangle$.$\} and its Hilbert A$-submodule $\mathcal{N} \subseteq \mathcal{M}$ over arbitrary $\mathrm{C}^{*}$-algebras $A$ (if there exist any such embeddings $\phi$ at all).

Proposition 3.3. Let $A$ be a $C^{*}$-algebra, $\{\mathcal{M}\langle.,\rangle$.$\} be a Hilbert A$-module and $\mathcal{N} \subseteq$ $\mathcal{M}$ be a Hilbert $A$-submodule. Suppose there is an isometric A-linear embedding $\phi$ of 
the Banach $A$-module $\mathcal{N}^{\prime}$ into the Banach $A$-module $\mathcal{M}^{\prime}$ which extends the standard isometric A-linear embedding i $: \mathcal{N} \rightarrow \mathcal{M}^{\prime},(\mathrm{i}(n)=\langle., n\rangle$ for $n \in \mathcal{N})$. Then $\phi$ has the property that for every $r \in \mathcal{N}^{\prime}$ the element $\phi(r) \in \mathcal{M}^{\prime}$ equals zero on $\mathcal{N}^{\perp} \subseteq \mathcal{M}$.

Proof. By Lemma 3.5 of 20 the map $\phi$ is uniquely defined by the condition that it extends the canonical embedding of $\mathcal{N}$ into $\mathcal{M}^{\prime}$. Therefore, if $A$ is a $\mathrm{W}^{*}$-algebra we can refer to [22, Prop. 3.6] for the properties of $\phi$, and the desired property can be obtained from there.

In case $A$ is an arbitrary $\mathrm{C}^{*}$-algebra we turn to the bidual situation and use the construction described in the preliminaries, i.e. switch from the two Hilbert $A$ modules $\mathcal{N} \subseteq \mathcal{M}$ to their canonically derived Hilbert $A^{* *}$-modules $\mathcal{N}^{\#} \subseteq \mathcal{M}^{\#}$. The isometric $A$-linear embedding i : $\mathcal{N} \hookrightarrow \mathcal{M}^{\prime}$ extends to an isometric $A^{* *}$-linear embedding i $\#$ of $\mathcal{N}^{\#}$ into the $A^{* *}$-dual Hilbert $A^{* *}$-module $\left(\mathcal{M}^{\#}\right)^{\prime}$ of $\mathcal{M}^{\#}$, cf. Proposition 2.3. Moreover, the isometric $A$-linear embedding $\phi: \mathcal{N}^{\prime} \hookrightarrow \mathcal{M}^{\prime}$ extends to an isometric $A^{* *}$-linear embedding $\phi:\left(\mathcal{N}^{\#}\right)^{\prime} \hookrightarrow\left(\mathcal{M}^{\#}\right)^{\prime}$ since there is a canonical chain of isometric $A$-linear embeddings $\phi\left(\mathcal{N}^{\prime}\right) \hookrightarrow \mathcal{M}^{\prime} \hookrightarrow\left(\mathcal{M}^{\#}\right)^{\prime}$ by [22] and, furthermore, every element of $\left(\mathcal{N}^{\#}\right)^{\prime}$ has a unique realization inside the biorthogonal complement $\phi\left(\mathcal{N}^{\prime}\right)^{\perp \perp}$ of the set $\phi\left(\mathcal{N}^{\prime}\right)$ calculated with respect to the Hilbert $A^{* *}$-module $\left(\mathcal{M}^{\#}\right)^{\prime}$. Now, part one of the proof applies and the restriction of every element $r^{\prime} \in \phi\left(\mathcal{N}^{\prime}\right)$ to $\mathcal{N}^{\perp} \subseteq \mathcal{M}$ turns out to be zero.

Going on, we make significant use of Lemma 3.2 to show Theorem 1.3. The example below ensures that the suppositions of Theorem 1.3 are in general weaker than those of the Theorems 1.1 and 1.2 .

Example 3.4. Consider the $\mathrm{C}^{*}$-algebra $A=l_{\infty}$, its two-sided ideal $I=c_{0}$ and the Hilbert $A$-module $\mathcal{M}=A \oplus I$ equipped with the $A$-valued inner product $\langle(a, i),(b, j)\rangle=a b^{*}+i j^{*}$ for $(a, i),(b, j) \in \mathcal{M}$. The Hilbert $A$-submodule $\mathcal{N}=\{(i, i): i \in I\}$ is a topological direct summand of $\mathcal{M}$ since $\mathcal{M}$ can be decomposed as $\mathcal{M}=\mathcal{N} \dot{+}\{(a, 0): a \in A\}$, but it is not an orthogonal direct summand. Moreover, $\mathcal{N} \equiv \mathcal{N}^{\perp \perp}$. The $A$-dual Hilbert $A$-module $\mathcal{N}^{\prime}=\{(a, a): a \in A\}$ can be boundedly and $A$-linearly embedded into the Hilbert $A$-module $\mathcal{M}^{\prime}=A \oplus A$ by the rule $\phi((a, a))(b, i):=2 i a^{*}$ for $a, b \in A, i \in I$. The restriction of $\phi\left(\mathcal{N}^{\prime}\right) \in \mathcal{M}^{\prime}$ to $\mathcal{N}^{\perp}=\{(-i, i): i \in I\} \subset \mathcal{M}$ is obviously non-zero, which contrasts with the assumptions (c) and (d) of Theorem 1.2 .

Proof of Theorem 1.3. We start with the proof of the implication (i) $\rightarrow$ (ii). Suppose $\mathcal{M}$ can be decomposed into the topological direct sum $\mathcal{M}=\mathcal{N} \dot{+} \tilde{\mathcal{N}}$. Since $\mathcal{N}$ is the kernel of a bounded $A$-linear idempotent operator it coincides with its biorthogonal complement $\mathcal{N}^{\perp \perp}$ inside $\mathcal{M}$, cf. Lemma 2.4. For every $r \in \mathcal{N}^{\prime}$, every $m \in \mathcal{M}$ the mapping $\phi: \mathcal{N}^{\prime} \rightarrow \mathcal{M}^{\prime}$ can be defined by the formula $\phi(r)[m]=$ $\phi(r)\left[n_{m}+\tilde{n}_{m}\right]:=r\left(n_{m}\right)$, so (ii) is fulfilled.

To show the converse conclusion (ii) $\rightarrow$ (i) consider an element $x \in \mathcal{M}^{\prime \prime} \stackrel{i}{\hookrightarrow} \mathcal{M}^{\prime}$. The element $x \in \mathcal{M}^{\prime \prime}$ applied to $\mathcal{M}^{\prime}$ defines a bounded $A$-linear functional on the subset $\phi\left(\mathcal{N}^{\prime}\right) \subseteq \mathcal{M}^{\prime}$ that belongs to $\mathcal{N}^{\prime \prime}$. By Lemma 3.2 the biorthogonal complement $\mathcal{N}^{\perp \perp}$ of $\mathcal{N}$ with respect to $\mathcal{M}^{\prime \prime}$ can be identified with $\mathcal{N}^{\prime \prime}$ preserving the canonical $A$-valued inner products. Therefore, there exists an element $y_{x} \in \mathcal{N}^{\prime \prime} \equiv \mathcal{N}^{\perp \perp} \subseteq \mathcal{M}^{\prime \prime}$ so that $\phi(r)\left[y_{x}\right]=\phi(r)[x]$ for any $r \in \mathcal{N}^{\prime}$. That is, $x=y_{x}+\left(x-y_{x}\right)$, and the summand $\left(x-y_{x}\right) \in \mathcal{M}^{\prime \prime}$ vanishes on $\phi\left(\mathcal{N}^{\prime}\right) \subseteq \mathcal{M}^{\prime}$. The map $x \in \mathcal{M}^{\prime \prime} \rightarrow y_{x} \in \mathcal{N}^{\prime \prime}$ is bounded and $A$-linear by the assumed properties of $\phi$. Consequently, $\mathcal{M}^{\prime \prime}$ admits a topologically direct sum decomposition as 
$\mathcal{M}^{\prime \prime}=\mathcal{N}^{\perp \perp} \dot{+} \tilde{\mathcal{N}}$, where $\tilde{\mathcal{N}}$ is the set $\left\{x \in \mathcal{M}^{\prime \prime}: \phi(r)[x]=0\right.$ for $\left.r \in \mathcal{N}^{\prime}\right\}$. This is condition (i).

The additional assumption of $\mathcal{M}$ being $A$-reflexive can in general not be dropped. V. M. Manuilov gave an example of a Hilbert $\mathrm{W}^{*}$-submodule $\mathcal{N}$ in a non-self-dual Hilbert $\mathrm{W}^{*}$-module $\mathcal{M}$ that coincides with its biorthogonal complement with respect to $\mathcal{M}$ and, nevertheless, is not a topological direct summand of $\mathcal{M}$. We realize that $\mathcal{N}$ additionally has the property (ii) required by Theorem 1.3 .

To be more precise, let $A=l_{\infty}$ be the $\mathrm{W}^{*}$-algebra of all bounded complex-valued sequences, $c_{0}$ be the ideal of all sequences converging to zero and select the Hilbert $A$-module $\mathcal{M}=l_{2}\left(c_{0}\right) \oplus l_{2}\left(l_{\infty}\right)$ with its standard $A$-valued inner product. Fix the injective operator $T \in \operatorname{End}_{A}\left(l_{2}\left(c_{0}\right)\right)$ defined by $T\left(\left\{a_{k}\right\}_{k}\right)=\left\{1 / k \cdot a_{k}\right\}_{k}$ and take the Hilbert $A$-submodule

$$
\mathcal{N}=\left\{(T(x), x): x \in l_{2}\left(c_{0}\right)\right\} \subset \mathcal{M} .
$$

Note, that $\mathcal{N}$ coincides with its biorthogonal complement $\mathcal{N}^{\perp \perp}$ with respect to $\mathcal{M}$. Since $\mathcal{M}^{\prime \prime}=\mathcal{M}^{\prime}=l_{2}\left(l_{\infty}\right)^{\prime} \oplus l_{2}\left(l_{\infty}\right)^{\prime}$ we have $\mathcal{N}^{\prime}=\left\{(T(x), x): x \in l_{2}\left(l_{\infty}\right)^{\prime}\right\} \hookrightarrow$ $\mathcal{M}^{\prime}$. The Hilbert $A$-submodule $\mathcal{N}$ can be realized as the kernel of a bounded nonadjointable module operator on $\mathcal{M}$.

However, $\mathcal{N}$ is not a topological direct summand of $\mathcal{M}$, whereas $\mathcal{N}^{\prime}$ is an orthogonal direct summand of $\mathcal{M}^{\prime}$. To see this, assume the existence of a topologically direct decomposition $\mathcal{M}=\mathcal{N}+\mathcal{L}$ for some Hilbert $A$-submodule $\mathcal{L}$ of $\mathcal{M}$. The resulting $A$-linear bounded idempotent operator $P: \mathcal{M} \rightarrow \mathcal{N}$ can be described by two operators $S_{1} \in \operatorname{End}_{A}\left(l_{2}\left(c_{0}\right)\right)$ and $S_{2} \in \operatorname{End}_{A}\left(l_{2}\left(l_{\infty}\right), l_{2}\left(c_{0}\right)\right)$ via the formula

$$
P: \mathcal{M} \rightarrow \mathcal{N} \quad, \quad P((x, y))=\left(T S_{1}(x)+T S_{2}(y), S_{1}(x)+S_{2}(y)\right) .
$$

Relying on the property $\left(\operatorname{id}_{\mathcal{M}}-P\right)^{2}=\left(\operatorname{id}_{\mathcal{M}}-P\right)$ we consider the action of $\left(\operatorname{id}_{\mathcal{M}}-P\right)$ on the special subsets $\left\{(0, y): y \in l_{2}\left(l_{\infty}\right)\right\}$ and $\left\{(x, 0): x \in l_{2}\left(c_{0}\right)\right\}$ of $\mathcal{M}$ and obtain the operator equalities

$$
\begin{aligned}
-T S_{2}+T S_{1} T S_{2}-T S_{2}\left(\mathrm{id}-S_{2}\right) & =-T S_{2}, \\
\mathrm{id}-T S_{1}-T S_{1}\left(\mathrm{id}-T S_{1}\right)+T S_{2} S_{1} & =\mathrm{id}-T S_{1} .
\end{aligned}
$$

By the injectivity of $T$ they can be transformed to the operator equalities

$$
\left(S_{1} T+S_{2}-\mathrm{id}\right) S_{2}=0 \quad, \quad\left(S_{1} T+S_{2}-\mathrm{id}\right) S_{1}=0
$$

which are valid on $l_{2}\left(l_{\infty}\right)$ and on $l_{2}\left(c_{0}\right)$, respectively. Consequently, the operator $\left(S_{1} T+S_{2}-\right.$ id $)$ equals zero on $l_{2}\left(c_{0}\right)=\operatorname{ran}\left(S_{1}+S_{2}\right)$ and hence, on its $A$-dual Hilbert $A$-module $l_{2}\left(l_{\infty}\right)^{\prime}$ by [22, Prop. 3.6]. However, $S_{2} \neq \mathrm{id}+S_{1} T$ on $l_{2}\left(l_{\infty}\right) \subset l_{2}\left(l_{\infty}\right)^{\prime}$ since equality would cause $S_{1}$ to be unbounded by the range restriction to $S_{2}$ and $T$. This contradicts the assumption of the existence of a bounded $A$-linear idempotent operator $P: \mathcal{M} \rightarrow \mathcal{N}$.

\section{The inner PRoduct ASPECTS of the PRoof}

To continue the proofs of Theorem 1.1 and 1.2 we have to take into account aspects of Hilbert $\mathrm{C}^{*}$-modules related to orthogonality of elements, and of $\mathrm{C}^{*}$ submodules. So we leave the sphere of considerations on the Banach $\mathrm{C}^{*}$-module level and deal with orthogonal direct sum decompositions and orthogonal complements.

First, we show that the statements (i) and (ii) of Theorem 1.1 are fulfilled for $\mathrm{C}^{*}$-algebras $A$ that possess a monotone complete multiplier $\mathrm{C}^{*}$-algebra $M(A)$. In 
particular, the result reproduces [34, Th. 4.1] for the special set of operator $\mathrm{C}^{*}$ modules that consists of all Hilbert $\mathrm{C}^{*}$-modules. However, the restriction to the Hilbert $\mathrm{C}^{*}$-modules enables us to enlarge the class of well-behaved (in the sense of a Hahn-Banach theorem) coefficient $\mathrm{C}^{*}$-algebras beyond the set of injective $\mathrm{C}^{*}$ algebras because this restriction rules out most of the existing more complicated operator $\mathrm{C}^{*}$-modules.

Proposition 4.1. (cf. 20, Th. 3.8]) Let $A$ be a $C^{*}$-algebra that possesses a monotone complete multiplier $C^{*}$-algebra $M(A)$. Let $\{\mathcal{M},\langle.,\rangle$.$\} be a Hilbert A-module$ and $\mathcal{N} \subseteq \mathcal{M}$ be a Hilbert $A$-submodule. Every bounded $A$-linear mapping $r: \mathcal{N} \rightarrow A$ can be continued to an A-linear bounded mapping $r^{\prime}: \mathcal{M} \rightarrow A$ with the same norm and the property $r^{\prime}\left(\mathcal{N}^{\perp}\right)=\{0\}$. In fact, such an extension is unique.

Proof. If $A$ does not contain an identity, then any Hilbert $A$-module $\mathcal{M}$ can be considered as a Hilbert $M(A)$-module preserving the $\mathrm{C}^{*}$-dual Hilbert $\mathrm{C}^{*}$-module $\mathcal{M}^{\prime}$ since the set $\{a x: a \in A, x \in \mathcal{M}\}$ is norm-dense inside $\mathcal{M}$ and $A$ is an essential two-sided ideal of $M(A)$. Consequently, Theorem 3.8 of [20] applies showing the existence of the claimed extension of $r$ with the stressed for properties.

Proposition 4.2. Let $A$ be a $C^{*}$-algebra which possesses a monotone complete multiplier $C^{*}$-algebra $M(A)$. Let $\{\mathcal{M},\langle.,\rangle$.$\} be a Hilbert A$-module and $\mathcal{N} \subseteq \mathcal{M}$ be a Hilbert $A$-submodule. Then the biorthogonal complement $\mathcal{N}^{\perp \perp}$ of $\mathcal{N}$ inside the A-bidual Hilbert A-module $\mathcal{M}^{\prime \prime}$ of $\mathcal{M}$ estimated with respect to the continued $A$-valued inner product is an orthogonal direct summand of $\mathcal{M}^{\prime \prime}$.

Proof. By Lemma 3.7 of 20 the $A$-valued inner product on $\mathcal{M}$ lifts to an $M(A)$ valued inner product on the $M(A)$-dual Banach $M(A)$-module $\mathcal{M}^{\prime}$ of $\mathcal{M}$, which is the $A$-dual Banach $A$-module of $\mathcal{M}$ at the same time and becomes a self-dual Hilbert $M(A)$-module this way. The biorthogonal complement $\mathcal{N}^{\perp \perp}$ of $\mathcal{N}$ (embedded into $\mathcal{M}^{\prime}$ in the canonical way) with respect to $\mathcal{M}^{\prime}$ is a self-dual Hilbert $M(A)$-submodule and direct summand of $\mathcal{M}^{\prime}$, [5, Th. 4.1] and [1, Th. 2.8] or [20, Prop. 3.10]. Because of self-duality, the Hilbert $M(A)$-modules $\mathcal{N}^{\perp \perp} \subseteq \mathcal{M}^{\prime}$ and $\mathcal{M}^{\prime}$ equal the $M(A)$ bidual Hilbert $M(A)$-modules of $\mathcal{N}$ and $\mathcal{M}$, respectively, and $\mathcal{N}^{\perp \perp} \subseteq \mathcal{M}^{\prime}$ is a direct summand of $\mathcal{M}^{\prime}$ by [20, Prop. 3.10] or [4, Th. 2.8].

If $A=M(A)$ then the monotone completeness of $A$ implies the coincidence of $\mathcal{L}^{\prime}$ and $\mathcal{L}^{\prime \prime}$ for any Hilbert $A$-module $\mathcal{L}$ by [5] or [20]. Additional difficulties appear if $A$ is non-unital because the $A$-bidual Hilbert $A$-module can be different from the $M(A)$-bidual Hilbert $M(A)$-module of a given Hilbert $A$-module, in general. So we describe the $A$-bidual Hilbert $A$-modules of $\mathcal{N}$ and $\mathcal{M}$ simply as the norm-closed linear hulls of the sets $\left\{a \cdot x: a \in A, x \in \mathcal{N}^{\perp \perp} \subseteq \mathcal{M}^{\prime}\right\}$ and $\left\{a \cdot x: a \in A, x \in \mathcal{M}^{\prime}\right\}$, respectively, cf. Lemma 2.1. The property of Hilbert $M(A)$-submodules of $\mathcal{M}^{\prime}$ of being a direct summand of $\mathcal{M}^{\prime}$ as well as orthogonal complements are respected by this selection process. Consequently, the biorthogonal complement $\mathcal{N}^{\perp \perp} \subseteq \mathcal{M}^{\prime \prime}$ of $\mathcal{N}$ inside $\mathcal{M}^{\prime \prime}$ is a direct summand of $\mathcal{M}^{\prime \prime}$.

To show the converse we should give some facts about $A$-reflexive Hilbert $\mathrm{C}^{*}$ modules over $\mathrm{C}^{*}$-algebras $A$ fulfilling assertion (ii) of Theorem 1.1: 
Proposition 4.3. Let $A$ be a $C^{*}$-algebra satisfying assertion (ii) of Theorem 1.1. Let $\left\{\mathcal{M},\langle.,\rangle_{\mathcal{M}}\right\}$ be an $A$-reflexive Hilbert $A$-module. Then $\mathcal{M}$ is orthogonally complementary and every bounded $A$-linear operator on $\mathcal{M}$ possesses an adjoint operator.

Proof. Let $\left\{\mathcal{L},\langle., .\rangle_{\mathcal{L}}\right\}$ be another Hilbert $A$-module containing $\mathcal{M}$ as a Banach $A$ submodule, i.e. housing a bicontinuously embedded copy of the Banach $A$-module $\mathcal{M}$. We show that both $\mathcal{M}$ and its biorthogonal complement $\mathcal{M}^{\perp \perp}$ with respect to $\mathcal{L}$ possess the same $A$-dual Banach $A$-module $\mathcal{M}^{\prime}$. Indeed, consider an element $r$ of the isometrically embedded copy of $\mathcal{M}^{\prime}$ inside $\mathcal{L}^{\prime}$ that exists by Theorem 1.1,(ii) and has the properties claimed there. Restrict $r$ to the submodule $\mathcal{M}^{\perp \perp} \subseteq \mathcal{L}$. We obtain an element $r^{\prime}$ of the isometrically embedded copy of $\left(\mathcal{M}^{\perp \perp}\right)^{\prime}$ inside $\mathcal{L}^{\prime}$. The difference $\left(r-r^{\prime}\right)$ vanishes on $\mathcal{M}$ since $r$ and $r^{\prime}$ coincide there, and it vanishes on $\mathcal{M}^{\perp} \subseteq \mathcal{L}$ by the supposed properties of the embedded $A$-dual Banach $A$-modules formulated at Theorem 1.1, (ii). By Lemma 2.4 the element $\left(r-r^{\prime}\right)$ has to vanish on $\mathcal{M}^{\perp \perp} \subseteq \mathcal{L}$, too. This proves our claim. Investigating the pair of Hilbert $A$-modules $\mathcal{M} \subseteq \mathcal{M}^{\perp \perp}$ we know that $\mathcal{M}$ is $A$-reflexive and that the Hilbert $A$-modules share the same $A$-dual Banach $A$-module. These facts together with Lemma 3.1 force them to coincide.

Consider an arbitrary element $x \in \mathcal{L}$ and the restriction of the $A$-valued bounded functional $\langle., x\rangle_{\mathcal{L}}$ to $\mathcal{M}$. By supposition condition (ii) of Theorem 1.1 holds. So there exists another $A$-valued bounded functional $r_{x} \in \mathcal{L}^{\prime}$ with the properties that $r_{x}$ restricted to $\mathcal{M} \subseteq \mathcal{L}$ coincides with $\langle., x\rangle_{\mathcal{L}}$ and that $r_{x}$ restricted to the orthogonal complement $\mathcal{M}^{\perp}$ of $\mathcal{M}$ inside $\mathcal{L}$ equals the zero mapping. We want to find a Hilbert $A$-module containing both $\mathcal{M}$ isometrically as a Hilbert $A$-submodule and a copy of $r_{x}$. Let us show that the value $\left\langle r_{x}, r_{x}\right\rangle$ has a meaning and belongs in fact to $A$.

For this aim consider the self-dual Hilbert $A^{* *}$-module $\left(\mathcal{L}^{\#}\right)^{\prime}$ which is constructed from $\mathcal{L}$ in the canonical way described in the preliminaries. The two bounded $A$ linear functionals $\langle., x\rangle_{\mathcal{L}}$ and $r_{x}$ can be continued to elements of $\left(\mathcal{L}^{\#}\right)^{\prime}$, cf. [22, 19]. The lifting of the $A$-valued inner product on $\mathcal{L}$ to an $A^{* *}$-valued inner product on $\left(\mathcal{L}^{\#}\right)^{\prime}$ allows to define inner product values for any two elements of $\mathcal{L}^{\prime} \subseteq\left(\mathcal{L}^{\#}\right)^{\prime}$, in particular for $\langle., x\rangle$ and $r_{x}$. The $A^{* *}$-valued functional $\left(\langle., x\rangle-r_{x}\right)$ vanishes on $\mathcal{M}$ by construction. The extension of $\left(\langle., x\rangle-r_{x}\right)$ from $\mathcal{L}$ to $\left(\mathcal{L}^{\#}\right)^{\prime}$ preserves this property and extends its validity to the biorthogonal complement of $\mathcal{M}$ taken with respect to the self-dual Hilbert $A^{* *}$-module $\left(\mathcal{L}^{\#}\right)^{\prime}$, cf. Lemma 2.4. However, by construction and by self-duality the biorthogonal complement of $\mathcal{M}$ taken with respect to the self-dual Hilbert $A^{* *}$-module $\left(\mathcal{L}^{\#}\right)^{\prime}$ contains $r_{x}, 22$, 4 . We obtain the equality

$$
0=\left\langle r_{x}, x-r_{x}\right\rangle_{\left(\mathcal{L}^{\#}\right)^{\prime}}=r_{x}(x)^{*}-\left\langle r_{x}, r_{x}\right\rangle_{\left(\mathcal{L}^{\#}\right)^{\prime}}
$$

applying the functional to the particular element $r_{x}$. In other words, the value $\left\langle r_{x}, r_{x}\right\rangle_{\left(\mathcal{L}^{\#}\right)^{\prime}}=\left\langle r_{x}, x\right\rangle_{\left(\mathcal{L}^{\#}\right)^{\prime}}=r_{x}(x)^{*}$ is an element of $A$. Consequently, the $A$ valued inner product on $\mathcal{M}$ can be continued to an $A$-valued inner product on the Banach $A$-submodule of $\mathcal{M}^{\prime}$ generated by $\mathcal{M}$ and $r_{x}$, reducing the $A^{* *}$-valued inner product on $\left(\mathcal{L}^{\#}\right)^{\prime}$ first to the embedded subset $\mathcal{M}^{\prime}$ and than to the Banach $A$-module of interest. That way the range of this inner product reduces to a subset of $A$, cf. [22, 19].

Now, by Lemma 3.1 the assumption $r_{x} \notin \mathcal{M} \hookrightarrow \mathcal{M}^{\prime}$ leads to a contradiction. Indeed, in this case we have constructed a Hilbert $A$-module containing $\mathcal{M}$ as a 
proper Hilbert $A$-submodule and possessing the same $A$-dual Banach $A$-module $\mathcal{M}^{\prime}$ by construction, which contradicts the supposed $A$-reflexivity of $\mathcal{M}$.

Summing up, for every element $x \in \mathcal{L}$ there exists an element $r_{x} \in \mathcal{M} \subseteq \mathcal{L}$ so that the orthogonal decomposition $x=r_{x} \oplus\left(x-r_{x}\right)$ holds inside $\mathcal{L}$. Hence, $\mathcal{M}$ is a direct summand of $\mathcal{L}$, and $\mathcal{M}$ is orthogonally complementary as a Hilbert $A$-module by definition. To see that any bounded module operator on $\mathcal{M}$ admits an adjoint consider $\mathcal{M}$ as a Hilbert $\langle\mathcal{M}, \mathcal{M}\rangle$-module and apply Proposition 2.2.

Proposition 4.4. Let $A$ be a $C^{*}$-algebra with property (ii) of Theorem 1.1. Then the multiplier $C^{*}$-algebra $M(A)$ of $A$ is monotone complete.

Proof. By [23, Th. 2.4] and Proposition 2.3 the $M(A)$-valued inner product of each Hilbert $M(A)$-module can be continued to an $M(A)$-valued inner product on its $M(A)$-bidual Banach $M(A)$-module. Since we want to show that our suppositions allow the extension to an $M(A)$-valued inner product on its $M(A)$-dual Banach $M(A)$-module we can suppose $M(A)$-reflexivity without loss of generality.

So let $\{\mathcal{M},\langle.,\rangle$.$\} be an M(A)$-reflexive Hilbert $M(A)$-module and consider the subset $\mathcal{N}=\{x \in \mathcal{M}:\langle x, x\rangle \in A\}$. The set $\mathcal{N}$ is an $A$-module. Indeed, the condition $\langle x, x\rangle \in A$ allows a representation of $x \in \mathcal{M}$ as $x=a y$ for some $a \in A$, $y \in \mathcal{M}$ by Lemma 2.1 and, therefore, for $x_{1}, x_{2} \in \mathcal{N}$ with $x_{i}=a_{i} y_{i}$ for $a_{i} \in A$, $y_{i} \in \mathcal{M}, i=1,2$ we obtain

$$
\begin{aligned}
& \left\langle x_{1}+x_{2}, x_{1}+x_{2}\right\rangle= \\
& \quad=\left\langle a_{1} y_{1}+a_{2} y_{2}, a_{1} y_{1}+a_{2} y_{2}\right\rangle \\
& \quad=a_{1}\left\langle y_{1}, y_{1}\right\rangle a_{1}^{*}+a_{2}\left\langle y_{2}, y_{1}\right\rangle a_{1}^{*}+a_{1}\left\langle y_{1}, y_{2}\right\rangle a_{2}^{*}+a_{2}\left\langle y_{2}, y_{2}\right\rangle a_{2}^{*} \in A .
\end{aligned}
$$

Hence, the $A$-module axioms are fulfilled by the rules for $\mathrm{C}^{*}$-valued inner products and ideals. Furthermore, $\mathcal{N}$ is complete with respect to the norm on $\mathcal{M}$.

We want to demonstrate that the $A$-dual Banach $A$-module $\mathcal{N}^{\prime}$ of $\mathcal{N}$ coincides with the $M(A)$-dual Banach $M(A)$-module $\mathcal{M}^{\prime}$ of $\mathcal{M}$. Note that any $M(A)$-linear map $r: \mathcal{N} \rightarrow M(A)$ takes its values in $A \subseteq M(A)$ by Lemma 2.1. First, suppose the restriction of two bounded module mappings $r_{1}, r_{2}: \mathcal{M} \rightarrow M(A)$ to $\mathcal{N}$ gives the same element of $\mathcal{N}^{\prime}$. Then the Hilbert $M(A)$-submodule $\left(\operatorname{ker}\left(r_{1}-r_{2}\right)\right)^{\perp} \subseteq \mathcal{M}$ does not contain any element from $\mathcal{N}$, i.e. for $x \in\left(\operatorname{ker}\left(r_{1}-r_{2}\right)\right)^{\perp}, x \neq 0$, we have $\langle x, x\rangle \in M(A) \backslash A$. We obtain a two-sided ideal $\left\langle\left(\operatorname{ker}\left(r_{1}-r_{2}\right)\right)^{\perp},\left(\operatorname{ker}\left(r_{1}-r_{2}\right)\right)^{\perp}\right\rangle \subseteq$ $M(A)$ possessing a trivial intersection with $A$ inside $M(A)$. The properties of a multiplier algebra imply $\left(\operatorname{ker}\left(r_{1}-r_{2}\right)\right)^{\perp}=\{0\}$ and $r_{1}=r_{2}$ on $\mathcal{M}$. Consequently, $\mathcal{M}^{\prime} \subseteq \mathcal{N}^{\prime}$. To deal with the second duals we have to use footnotes to distinguish between the ranges of the $\mathrm{C}^{*}$-linear functionals on $\mathcal{N}$. Since $\mathcal{M}_{M(A)}^{\prime} \subseteq \mathcal{N}_{M(A)}^{\prime}$ we get

$$
\mathcal{M} \equiv \mathcal{M}_{M(A)}^{\prime \prime} \supseteq \mathcal{N}_{M(A)}^{\prime \prime} \supseteq \mathcal{N}_{A}^{\prime \prime} \supseteq \mathcal{N} .
$$

Since $\mathcal{N}$ is the largest submodule of $\mathcal{M}$ taking inner product values in $A$ and 23 , Th. 2.4] guarantees the same property for the Hilbert $A$-submodule $\mathcal{N}_{A}^{\prime \prime}$ of $\mathcal{M}$ both they have to coincide, and $\mathcal{N}$ turns out to be $A$-reflexive.

Now, the assumption to $A$ to fulfil condition (ii) of Theorem 1.1 comes into the play. By Proposition $4.3 \mathcal{N}$ is orthogonally complementary as a Hilbert $A$ module. In particular, setting $\mathcal{M}=M(A)$ we obtain that $\mathcal{N}=A$ is orthogonally complementary as a Hilbert $A$-module, too, and that the $A$-dual Banach $A$-module of $A$ that coincides with the set of all left multipliers of $A$ in fact can be identified 
with $M(A)$ by Proposition 2.2. Referring again to Proposition 2.2 we establish that the $A$-valued inner product on $\mathcal{N} \oplus A$ can be continued to an $M(A)$-valued inner product on $\mathcal{N}^{\prime} \oplus M(A) \equiv \mathcal{M}^{\prime} \oplus M(A)$ turning $\mathcal{M}^{\prime} \oplus M(A)$ into a self-dual Hilbert $M(A)$-module. So $\mathcal{M}^{\prime}$ is a self-dual Hilbert $M(A)$-module, too.

Summarizing, the $M(A)$-valued inner product of every Hilbert $M(A)$-module can be canonically continued to an $M(A)$-valued inner product on its $M(A)$-bidual Banach $M(A)$-module by [23, Th. 2.4] and, what is more, on its $M(A)$-dual Hilbert $M(A)$-module. Referring to Proposition 2.3 we are done.

Proposition 4.5. Let $A$ be a $C^{*}$-algebra, $\{\mathcal{M},\langle.,\rangle$.$\} be a Hilbert A-module and$ $\mathcal{N} \subseteq \mathcal{M}$ be its Hilbert $A$-submodule. Suppose the pair $\{\mathcal{M}, \mathcal{N}\}$ fulfills the suppositions of Proposition 3.5. Then the A-bidual Hilbert $A$-module $\mathcal{M}^{\prime \prime}$ of $\mathcal{M}$ decomposes into a direct orthogonal sum as $\mathcal{M}^{\prime \prime}=\mathcal{N}^{\perp \perp} \oplus \mathcal{N}^{\perp}$, and the A-bidual Banach $A$ modules $\mathcal{N}^{\prime \prime}$ and $\left(\mathcal{N}^{\perp \perp}\right)^{\prime \prime}$ coincide. The converse conclusion is also true. Both the Hilbert $A$-submodules $\mathcal{N}$ and $\mathcal{N}^{\perp \perp} \subseteq \mathcal{M}$ are not in general direct summands of $\mathcal{M}$ or $\mathcal{M}^{\prime \prime}$.

Proof. The $A$-valued inner product on $\mathcal{M}$ can be continued to an $A$-valued inner product on the $A$-bidual Banach $A$-module $\mathcal{M}^{\prime \prime}$ of $\mathcal{M}$ by [23, Th. 2.4]. Moreover, the biorthogonal complement $\mathcal{N}^{\perp \perp}$ of $\mathcal{N}$ with respect to $\mathcal{M}^{\prime \prime}$ is isomorphic to the $A$-bidual Banach $A$-module $\left(\mathcal{N}^{\perp \perp}\right)^{\prime \prime}$ by Lemma 3.1.

A discussion similar to that in the proof of Proposition 4.3 shows that every element $\langle., m\rangle \in \mathcal{M}^{\prime}$ with $m \in \mathcal{M}^{\prime \prime}$ can be decomposed into the direct sum of an element $r_{m} \in \mathcal{N}^{\perp \perp}$ and an element $\left(\langle., m\rangle-r_{m}\right) \in \mathcal{N}^{\perp}$. Consequently, $\mathcal{M}^{\prime \prime}=\mathcal{N}^{\perp \perp} \oplus \mathcal{N}^{\perp}$ implying $\mathcal{M}^{\prime}=\left(\mathcal{N}^{\perp \perp}\right)^{\prime} \oplus\left(\mathcal{N}^{\perp}\right)^{\prime}$ and $\left(\mathcal{N}^{\perp \perp}\right)^{\prime} \equiv \mathcal{N}^{\prime}$ by the supposed property (ii) of Theorem 1.2. Refering to Lemma 3.1 we obtain the identity $\left(\mathcal{N}^{\perp \perp}\right)^{\prime \prime} \equiv \mathcal{N}^{\prime \prime}$

The second assertion on the converse conclusion follows from Lemma 3.1 if we continue bounded module mappings $r \in\left(\mathcal{N}^{\perp \perp}\right)^{\prime}$ to bounded module mappings $r^{\prime} \in \mathcal{M}^{\prime}$ by simply setting $r^{\prime}=r \circ P$, where $P: \mathcal{M}^{\prime \prime} \rightarrow \mathcal{N}^{\perp \perp}$ is the projection existing by assumption.

To give an example of a situation where $\mathcal{N}$ is not a direct summand of $\mathcal{M} \equiv \mathcal{M}^{\prime \prime}$, but its biorthogonal complement $\mathcal{N}^{\perp \perp}$ is, consider the $\mathrm{W}^{*}$-algebra $A=\mathcal{M}$ of all bounded linear operators on a separable Hilbert space as a Hilbert $A$-module over itself and set $I=\mathcal{N}$ to be the Hilbert $A$-submodule of all compact operators. For this pair the generalized Hahn-Banach theorem holds.

As an example for the case $\mathcal{M} \not \equiv \mathcal{M}^{\prime \prime}$ consider the Hilbert $A$-module $\mathcal{M}=A \oplus I$ equipped with its canonical $A$-valued inner product for the same $\mathrm{W}^{*}$-algebra $A$. The Hilbert $A$-submodule $\mathcal{N}=\{(i, i): i \in I\}$ coincides with its biorthogonal complement taken with respect to $\mathcal{M}$, but it is not an orthogonal direct summand of $\mathcal{M}$. By contrast its biorthogonal complement $\mathcal{N}^{\perp \perp}=\{(a, a): a \in A\}$ in the $A$-bidual Hilbert $A$-module $\mathcal{M}^{\prime \prime}=A \oplus A$ of $\mathcal{M}$ certainly is an orthogonal direct summand.

The following example shows that the bidual Banach $\mathrm{C}^{*}$-modules of a Hilbert $\mathrm{C}^{*}$-submodule and of its biorthogonal complement do not in general coincide: if we set $A=\mathcal{M}=\mathrm{C}([0,1])$ and $\mathcal{N}=\mathrm{C}_{0}((0,1])$, then $\mathcal{M}=\mathcal{N}^{\perp \perp} \subseteq \mathcal{M}$, but the Hahn-Banach theorem does not hold for the pair $\{\mathcal{N}, \mathcal{M}\}$ of Hilbert $A$-modules (see introduction) since both $\mathcal{N}^{\perp \perp} \equiv \mathcal{M}$ and $\mathcal{N}$ are $A$-reflexive, but different. 
Proof of Theorem 1.1 And 1.2. Proposition 3.3 demonstrates that the first set of conditions stated at Theorem 1.2 implies the second one. To show the converse implication consider two elements $r_{1}, r_{2} \in \mathcal{N}^{\prime}$, an element $a \in A$ and the extended to $\mathcal{M}^{\prime}$ elements $s:=\left(r_{1}+r_{2}\right)^{\prime}-r_{1}^{\prime}-r_{2}^{\prime}, t:=a\left(r^{\prime}\right)-(a r)^{\prime}$. By assumption $s, t \in \mathcal{M}^{\prime}$ vanish on both $\mathcal{N}$ and on $\mathcal{N}^{\perp} \subseteq \mathcal{M}$. Since the orthogonal complement of $\mathcal{N} \oplus \mathcal{N}^{\perp}$ with respect to $\mathcal{M}$ is trivial Lemma 2.4 applies, and $s=t=0$ on $\mathcal{M}$. So by assumption the subset of $\mathcal{M}^{\prime}$ consisting of all extended elements of $\mathcal{N}^{\prime}$ has the structure of a Banach $A$-module inherited from that of the Banach $A$-module $\mathcal{N}^{\prime}$. Moreover, for $x \in \mathcal{N}$ the element $\left(\langle., x\rangle-(\langle., x\rangle)^{\prime}\right) \in \mathcal{M}^{\prime}$ vanishes on both $\mathcal{N}$ and $\mathcal{N}^{\perp}$, too, by assumption. The same argument as above yields $\langle., x\rangle \equiv(\langle., x\rangle)^{\prime}$ for any $x \in \mathcal{N}$.

By the way we have obtained the equivalence of the assertions (i) and (ii) of Theorem 1.1. Together with the Propositions 4.1, 4.4, 4.2, 4.5 and 2.3 the proofs of Theorem 1.1 and 1.2 are complete.

\section{Final REMARKS}

Hahn-Banach type problems for Hilbert $\mathrm{C}^{*}$-modules are closely related to those for general operator modules as treated by G. Vincent-Smith 31, G. Wittstock 33, 34, Ching-Yun Suen 30, P. S. Muhly and Qiyuan Na [21, A. M. Sinclair and R. R. Smith 29, and others. Hilbert $\mathrm{C}^{*}$-modules serve as a special class of examples of operator modules, [34, Prop. 2.7, 2.8]. All these Hahn-Banach type theorems for completely bounded module maps on matricial normed Banach $\mathrm{C}^{*}$ (bi-)modules (i.e. operator (bi-)modules over $\mathrm{C}^{*}$-algebras) are formulated under the assumption that the the range of the extensions is allowed to grow inside a (larger) injective $\mathrm{C}^{*}$-algebra, for example the injective envelope of the $\mathrm{C}^{*}$-algebra of coefficients $([12])$. Naturally, order aspects play a mayor role in these investigations, cf. [21]. All these theorems give only sparse information about situations with sharper codomain restrictions, so Hilbert $\mathrm{C}^{*}$-modules could serve as a model to develop more general Hahn-Banach type criteria for operator (bi-)modules. Our presented case study demonstrates that monotone complete $\mathrm{C}^{*}$-algebras serve as an upper bound for attempts to extend these results to a larger class of $\mathrm{C}^{*}$-algebras of coefficients and codomains. However, the sharpest restriction has been obtained in collaboration with V. I. Paulsen during a research stay of the author in Houston in 1998. The example belongs to another class of operator bimodules: taking an arbitrary $\mathrm{C}^{*}$-algebra $A$ and its injective envelope $I(A)$, a monotone complete $\mathrm{C}^{*}$-algebra $([12])$, so this pair of operator $A$-bimodules fulfills a Hahn-Banach type extension theorem for completely bounded $A$-linear maps into $A$ if and only if the $\mathrm{C}^{*}$-algebra $A$ is injective itself (and coincides with its injective envelope). So the Wittstock extension theorems for operator (bi-)modules turn out to be actually already criteria. Furthermore, after a talk of the author given at a meeting on operator spaces at CIRM in Marseille in July 1998 M. Junge formulated the idea that for an arbitrary pair of an operator bimodule and one of its operator subbimodules over a given $\mathrm{C}^{*}$-algebra $A$ any completely bounded module map of the submodule to $A$ admits a completely bounded extension to the entire operator bimodule preserving the completely boundedness norm and taking values at most in the bidual to $A \mathrm{~W}^{*}$-algebra $A^{* *}$, if and only if $A$ has the weak expectation property in the sense of E. C. Lance [17]. The proof was found during discussions of M. Junge with 
the author at that meeting. These results will be published elsewhere since they lead beyond the topic of the presented paper, see [10], [11.

As a second addition we want to explain another equivalent condition to the assertions of Theorem 1.1 which is much more technical. We get an idea of how particular mappings $r \in \mathcal{N}^{\prime}$ may be isometrically extended to mappings $r^{\prime} \in \mathcal{M}^{\prime}$ failing to be zero on $\mathcal{N}^{\perp} \subseteq \mathcal{M}$ (cf. Example 3.4):

Proposition 5.1. Let $A$ be a $C^{*}$-algebra with the property that for every Hilbert $A$ module $\mathcal{M}$ and every Hilbert $A$-submodule $\mathcal{N} \subseteq \mathcal{M}$ every bounded $A$-linear mapping $r: \mathcal{N} \rightarrow A$ can be continued to a bounded A-linear mapping $r^{\prime}: \mathcal{M} \rightarrow A$ possessing the same norm so that the restriction of $r^{\prime}$ to $\mathcal{N}$ equals $r$. If additionally the equality $\langle r, r\rangle=\left\langle r^{\prime}, r^{\prime}\right\rangle \in A^{* *}$ is supposed to hold for the $A^{* *}$-valued inner product on the self-dual Hilbert $A^{* *}$-module $\left(\mathcal{M}^{\#}\right)^{\prime}$, then the restriction of each extension $r^{\prime}$ to $\mathcal{N}^{\perp} \subseteq \mathcal{M}$ equals zero and hence, $A$ has the property (ii) of Theorem 1.1.

Proof. The mapping $r^{\prime}: \mathcal{M} \rightarrow A$ canonically continues to an element of $\left(\mathcal{M}^{\#}\right)^{\prime}$ and possesses a canonical orthogonal decomposition $r^{\prime}=r_{1} \oplus r_{2}$ with $r_{1}, r_{2} \in\left(\mathcal{M}^{\#}\right)^{\prime}, r_{1}$ restricted to $\left(\mathcal{N}^{\#}\right)^{\perp} \subseteq\left(\mathcal{M}^{\#}\right)^{\prime}$ equals zero and $r_{2}$ restricted to $\mathcal{N}^{\#} \subseteq\left(\mathcal{M}^{\#}\right)^{\prime}$ equals zero, too. The Banach $A$-module $\mathcal{N}^{\prime}$ is canonically and isometrically embedded into $\left(\mathcal{N}^{\#}\right)^{\prime} \equiv\left(\mathcal{N}^{\#}\right)^{\perp \perp} \subseteq\left(\mathcal{M}^{\#}\right)^{\prime}$. Consequently, $r=r_{1}$ and the equality

$$
\left\langle r^{\prime}, r^{\prime}\right\rangle=\langle r, r\rangle+\left\langle r_{2}, r_{2}\right\rangle \in A^{* *}
$$

holds for the appropriate values of the $A^{* *}$-valued inner product on $\left(\mathcal{M}^{\#}\right)^{\prime}$. Hence, $r_{2}$ has to be zero to fulfill the additional assumption.

The point is that the metric equality $\|r\|=\left\|r^{\prime}\right\|=\left\|\langle r, r\rangle+\left\langle r_{2}, r_{2}\right\rangle\right\|_{A}^{1 / 2}$ can in general be valid for (existing) extensions $r^{\prime}$ of $r$ even if $r_{2} \neq 0$. We have to conclude that the consideration of any possible extensions $r^{\prime} \in \mathcal{M}^{\prime}$ of a given bounded $A$ linear mapping $r: \mathcal{N} \rightarrow A$ (if there is any at all) has to be made separately in every particular situation.

For example, consider the $\mathrm{C}^{*}$-algebra $A=\mathrm{C}([0,1])$ of all continuous functions on the unit interval $[0,1]$ as a Hilbert $A$-module $\mathcal{M}$ over itself. Set $\mathcal{N}=\{f \in A$ : $f \equiv 0$ on $[1 / 2,1]\}$. The embedding map i $: \mathcal{N} \rightarrow A$ is an element of $\mathcal{N}^{\prime}$ which does not belong to the subset $\{\langle., n\rangle: n \in \mathcal{N}\} \subset \mathcal{N}^{\prime}$. Every function $f_{i} \in A$ taking the value 1 on the interval $[0,1 / 2]$ and satisfying the equality $\left\|f_{i}\right\|=1$ is an extension of $\mathrm{i} \in \mathcal{N}^{\prime}$ inside $\mathcal{M}^{\prime}=A$. However, $\mathcal{N}^{\perp \perp}$ is not a direct summand of $A=\mathcal{M}$ and there does not exist any extension $f_{i}$ of i in $\mathcal{M}$ whose restriction to $\mathcal{N}^{\perp}$ equals zero.

Problem 5.2. Denote by $\mathcal{M}$ and $\mathcal{N} \subseteq \mathcal{M}$ a Hilbert $C^{*}$-module and one of its Hilbert $C^{*}$-submodules. For which kind of $C^{*}$-algebras $A$ is the biorthogonal complement $\mathcal{N}^{\perp \perp}$ of $\mathcal{N}$ inside $\mathcal{M}$ or inside its A-bidual Hilbert A-module $\mathcal{M}^{\prime \prime}$, respectively, always a topological or orthogonal direct summand?

Acknowledgement: The author thanks D. P. Blecher, K.-D. Kürsten, V. M. Manuilov, V. I. Paulsen and E. V. Troitsky for valuable discussions and comments.

\section{REFERENCES}

[1] M. Baillet, Y. Denizeau, J.-F. Havet, Indice d'une esperance conditionelle, Compos. Math. 66(1988), 199-236.

[2] D. P. Blecher, A generalization of Hilbert modules, J. Funct. Anal. 136(1996), 365-421. 
[3] E. E. Floyd, Boolean algebras with pathological order topologies, Pacific J. Math. 5(1955), 687-689.

[4] M. Frank, Self-duality and C*-reflexivity of Hilbert C*-modules, Zeitschr. Anal. Anwendungen 9(1990), 165-176.

[5] M. Frank, Hilbert $\mathrm{C}^{*}$-modules over monotone complete $\mathrm{C}^{*}$-algebras, Math. Nachrichten 175(1995), 61-83.

[6] M. Frank, Beiträge zur Theorie der Hilbert- $C^{*}$-Moduln, Habilitation thesis, Universität Leipzig, 1997; Shaker Verlag, Aachen, 1997.

[7] M. Frank, A multiplier approach to the Lance-Blecher theorem, Zeitschr. Anal. Anwendungen 16(1997), 565-573.

[8] M. Frank, Isomorphisms of Hilbert $\mathrm{C}^{*}$-modules and *-isomorphisms of related operator C*-algebras, Math. Scand. 80(1998), 313-319.

[9] M. Frank, Geometrical aspects of Hilbert C*-modules, Positivity 3(1999), 215-243.

[10] M. Frank, V. I. PAUlsen, Injective envelopes of $\mathrm{C}^{*}$-algebras as operator modules, preprint, University of Houston, Houston, TX, U.S.A. and Universität Leipzig, Leipzig, Germany, 1999.

[11] M. Frank, Hahn-Banach theorems for operator modules, and the weak expectation property, in preparation, Universität Leipzig, Leipzig, Germany, 1999.

[12] M. Hamana, Injective envelopes of C*-algebras, J. Math. Soc. Japan 31(1979), 181-197.

[13] M. Hamana, Modules over monotone complete $\mathrm{C}^{*}$-algebras, Internat. J. Math. 3(1992), 185-204.

[14] K. K. Jensen, K. Thomsen, Elements of KK-Theory, (Series: Mathematics: Theory \& Applications), Birkhäuser, Boston-Basel-Berlin, 1991.

[15] I. KAPlansky, Projections in Banach algebras, Ann. Math. 53(1951), 235-249.

[16] G. G. Kasparov, Hilbert $\mathrm{C}^{*}$-modules: The theorems of Stinespring and Voiculescu, J. Operator Theory 4(1980), 133-150.

[17] E. C. LANCE, On nuclear C*-algebras, J. Funct. Anal. 12(1973), 157-176.

[18] E. C. LANCE, Hilbert $C^{*}$-modules - a toolkit for operator algebraists, London Mathematical Society Lecture Note Series 210, Cambridge University Press, Cambridge, England, 1995.

[19] H. Lin, Bounded module maps and pure completely positive maps, J. Operator Theory 26(1991), 121-138.

[20] H. Lin, Injective Hilbert C*-modules, Pac. J. Math. 154(1992), 131-164.

[21] P. S. Muhly, QIYuan Na, Extensions of completely bounded $A-B$ bimodule maps, Glasgow Math. J. 36(1994), 145-155.

[22] W. L. PASchke, Inner product modules over B*-algebras, Trans. Amer. Math. Soc. 182(1973), 443-468.

[23] W. L. PAschke, The double B-dual of an inner product module over a $\mathrm{C}^{*}$-algebra B, Canad. J. Math. 26(1974), 1272-1280.

[24] G. K. Pedersen, Multipliers in AW*-algebras, Math. Z. 187(1984), 23-24.

[25] G. K. Pedersen, SAW*-algebras and corona $\mathrm{C}^{*}$-algebras, contributions to non-commutative topology, J. Oper. Theory 15(1986), 15-32.

[26] G. K. Pedersen, Factorization in C*-algebras, Expo. Math. 16(1998), 145-156.

[27] I. Raeburn and D. P. Williams, Morita Equivalence and Continuous Trace $C^{*}$-algebras, Math. Surveys and Monogr. v. 60, Amer. Math. Soc., Providence, R.I., 1998.

[28] K. Saitô, J. D. Maitland Wright, All AW*-factors are normal, J. London Math. Soc.(2) 44(1991), 143-154.

[29] A. M. Sinclair, R. R. Smith, Factorization of completely bounded bilinear operators and injectivity, J. Funct. Anal. 157(1998), 62-87.

[30] Ching-Yun Suen, Completely bounded maps on $\mathrm{C}^{*}$-algebras, Proc. Amer. Math. Soc. 93(1985), 81-87.

[31] G. Vincent-Smith, The Hahn-Banach theorem for modules, Proc. London Math. Soc. 17(1967), 72-90.

[32] N. E. WegGe-Olsen, K-theory and $C^{*}$-algebras - a friendly approach, Oxford University Press, Oxford, 1993.

[33] G. Wiтtsтock, Ein operatorwertiger Hahn-Banach Satz, J. Functional Analysis 40(1981), $127-150$

[34] G. Wittstock, Extensions of completely bounded C*-module homomorphisms, Proc. Conf. on Operator Algebras and Group Representations, Neptun, 1980, Pitman, 1983, 238-250. 
Universität Leipzig, Mathematisches Institut, Augustusplatz 10, D-04109 Leipzig, Fed. Rep. Germany, Frank@mathematik.uni-LeipZig.de 